\title{
The Nuclear Quadrupole Resonance Magnetometer: A New Method for the Precision Measurement of the Magnitude and Direction of Magnetic Fields
}

\author{
Melvin Linzer \\ Institute for Materials Research, National Bureau of Standards, Washington, D.C. 20234
}

(September 11, 1969)

\begin{abstract}
A theoretical investigation of the feasibility of a magnetometer based on the observation of the Zeeman splitting of a nuclear quadrupole resonance line is presented. With a single crystal of potassium chlorate as the sensor, an nqr magnetometer with center frequency of $28 \mathrm{MHz}$ and bandwidth of $10 \mathrm{MHz}$ could cover a range of $10 \mathrm{nT}$ to greater than $1 \mathrm{~T}$. Precision at specific crystal orientations should approach $10 \mathrm{nT}$ in the magnitude of the field, at flux densities exceeding $100 \mu \mathrm{T}$, and $(65 / B)$ microradians in the direction of the field where $B$ is the flux density in $\mathrm{mT}$. If circularly polarized $\mathrm{rf}$ excitation is employed, a precision of $10 \mathrm{nT}$ could be achieved at flux densities less than $100 \mu \mathrm{T}$. Accuracy of the flux density measurements is limited by the uncertainty in the gyromagnetic ratio of ${ }^{35} \mathrm{Cl}$, which at the present time is approximately $1 \times 10^{-4}$. The effect of the small asymmetry parameter which characterizes the ${ }^{35} \mathrm{Cl}$ resonance in $\mathrm{KClO}_{3}$ is analyzed. Various experimental arrangements are proposed to record the magnitude and direction of the field, to obtain high resolution at low fields and good linearity at high fields, to overcome the difficulties due to the presence of a small asymmetry parameter, and to make an instantaneous measurement of the Zeeman splitting frequency.
\end{abstract}

Key words: Magnetometer; nuclear quadrupole resonance.

\section{Basic Theory}

This paper presents a theoretical investigation of the feasibility of a magnetometer based on the observation of the Zeeman splitting of a nuclear quadrupole resonance (nqr) line. A detailed treatment of the Zeeman effect can be found in the monograph of Das and Hahn $[1],{ }^{1}$ in the papers quoted therein, and in several subsequent publications [2-8]. We shall confine ourselves in this section to a discussion of the theory relevant to Zeeman splittings in single crystals and its application to the measurement of the magnitude and direction of magnetic fields. The nuclei will be assumed to be characterized by an asymmetry parameter, $\eta$, equal to zero. Moreover, the quadrupole energy will be considered sufficiently greater than the Zeeman energy such that the Zeeman splitting is essentially linear with respect to the magnetic field.

Special emphasis will be placed on the $|1 / 2| \leftrightarrow|3 / 2|$ transitions; these are responsible for the ${ }^{35} \mathrm{Cl}$ nqr spectrum in potassium chlorate $\left(\mathrm{KClO}_{3}\right)$, which, as will be shown later, is very suitable for magnetic field measurements. The effect of the small asymmetry parameter in $\mathrm{KClO}_{3}$ is discussed in the next section.

\footnotetext{
${ }^{1}$ Figures in brackets indicate the literature references at the end of this paper.
}

Transitions between the $\pm m$ and $\pm(m+1)$ levels, with $m \geqslant 1$, give rise to two frequencies spaced symmetrically about the pure quadrupole frequency, $\nu_{Q}$, with separation

$$
\begin{aligned}
\Delta \nu & =2 \nu_{L} \cos \theta \\
\nu_{L} & =\frac{|\gamma| B_{0}}{2 \pi}
\end{aligned}
$$

where $\nu_{L}$ is the Larmor frequency, $|\gamma|$ is the absolute value of the gyromagnetic ratio, $B_{0}$ is the magnetic flux density, and $\theta$ is the angle between the magnetic field and the symmetry axis of the electric field gradient [1]. The intensities of the two lines are onehalf the intensity of the original line and are independent of the orientation of the magnetic field.

For transitions between $\pm 1 / 2$ and $\pm 3 / 2$ levels, two pairs of splittings are in general observed because of the zeroth-order mixing of the $\pm 1 / 2$ states in the presence of a magnetic field. ${ }^{2}$ The energy level diagram is shown in figure 1 for the case applicable to the ${ }^{35} \mathrm{Cl}$ spectrum in $\mathrm{KClO}_{3}$. When $\eta=0$ and $\nu_{L} \rightarrow 0$,

\footnotetext{
${ }^{2}$ For convenience, we have labeled the four quantum states as $\pm 1 / 2, \pm 3 / 2$. The conventional designation of the $\pm 1 / 2$ levels in the presence of a small magnetic field is " \pm "; at flux densities sufficiently great to cause an appreciable admixture of all the levels, a dif ferent notation is usually employed [9].
} 


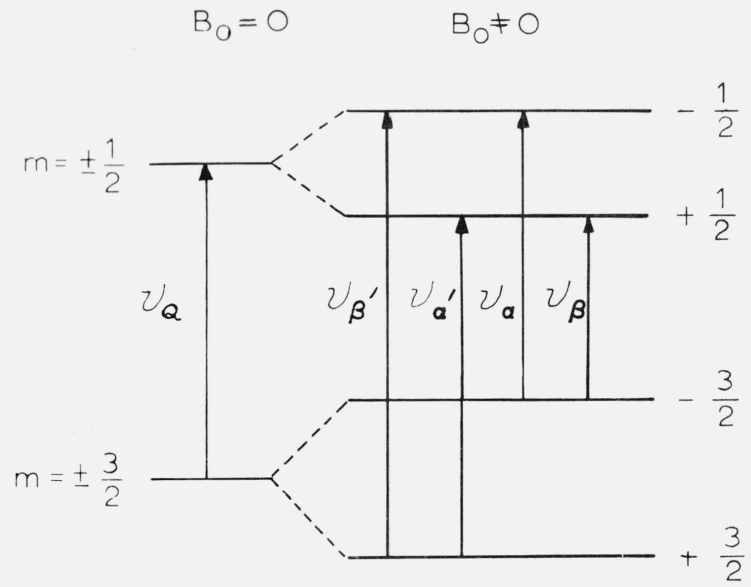

FIGURE 1. Low-field energy level diagram for the $|1 / 2| \leftrightarrow|3 / 2|$ transitions of a nucleus with half-integral spin, negative quadrupole coupling and positive gyromagnetic ratio.

The use of the quantum numbers $\pm m$ to designate the states in the case $B_{0} \neq 0$ is for convenience only.

the splittings are symmetrical about the pure quadrupole frequency, with the inner pair of lines, denoted by $\alpha \alpha^{\prime}$, always equal to or stronger in intensity than the outer pair of lines, denoted by $\beta \beta^{\prime}$. The splittings and relative intensities are given by the equations [1]:

$$
\begin{gathered}
\Delta \nu_{\alpha \alpha^{\prime}}=|3-f| \nu_{L} \cos \theta, I_{\alpha \alpha^{\prime}}=(f+1) / 2 f \\
\Delta \nu_{\beta \beta^{\prime}}=(3+f) \nu_{L} \cos \theta, I_{\beta \beta^{\prime}}=(f-1) / 2 f \\
f \equiv\left[1+(I+1 / 2)^{2} \tan ^{2} \theta\right]^{1 / 2}
\end{gathered}
$$

and are shown in figures 2 and 3 , respectively. The $\alpha$ components correspond to the transitions $\pm 1 / 2 \leftrightarrow \pm 3 / 2$, whereas the $\beta$ components arise from the transitions $\mp 1 / 2 \leftrightarrow \pm 3 / 2$.

Simplified spectra are obtained at three special orientations of the magnetic field. At $\theta=0^{\circ}$, only the splitting of the $\alpha$ components is observed with
$\Delta \nu_{\alpha \alpha^{\prime}}=2 \nu_{L}$. The $\beta$ components give rise to twice this splitting but have zero intensity at this orientation. At $\theta=90^{\circ}$, the $\alpha$ and $\beta$ components have identical intensities and splittings, with $\Delta \nu_{\alpha \alpha^{\prime}}=\Delta \nu_{\beta \beta^{\prime}}=(I+1 / 2) \nu_{L}$. At $\theta=\tan ^{-1}[2 \sqrt{2} /(I+1 / 2)]$, the splittings are given by

$$
\begin{aligned}
\Delta \nu_{\alpha \alpha^{\prime}} & =0 \\
\Delta \nu_{\beta \beta^{\prime}} & =6(I+1 / 2) \nu_{L} /\left[8+(I+1 / 2)^{2}\right]^{1 / 2} .
\end{aligned}
$$

For $I=3 / 2, \theta$ and $\Delta \nu_{\beta \beta^{\prime}}$ are equal to $54^{\circ} 44^{\prime}$ (one-half of the tetrahedral angle) and $2 \sqrt{3} \nu_{L}$, respectively. The separation between $\alpha$ and $\beta$ components is therefore $\sqrt{3} \nu_{L}$ at this orientation.

Because of their simplified spectral patterns, these special orientations are easily obtained experimentally. All three alinements give comparable line separations, with maximum splittings between adjacent lines appearing at $\theta=0^{\circ}$ and $90^{\circ}$.

Deviations from linearity with respect to the magnitude of the magnetic field depend very strongly on the orientation. of the symmetry axis. These deviations may be expressed in terms of a power series [9] in $\xi$, where $\xi=\nu_{L} / \nu_{Q}$ is the ratio of the Larmor frequency to the pure quadrupole frequency and is considered to be much less than one. At $\theta=0^{\circ}$, both the $\alpha$ and (vanishing) $\beta$ component splittings are exactly linear with the field. For the $\theta=54^{\circ} 44^{\prime}$ configuration, the $\beta$ splitting is approximately equal to $2 \sqrt{3}\left(1-\xi^{2} / 3\right)$ but the composite $\alpha$ line is merely broadened by $\sim \xi^{4}$. At $\theta=90^{\circ}$, the fractional deviation is $-3 / 2 \xi^{2}$ for the $\alpha$ splitting and zero for the $\beta$ splitting.

The Zeeman splittings of the lines are least sensitive to angle at $\theta=0^{\circ}$ and most sensitive at $\theta=90^{\circ}$. Inspection of figure 2 reveals several large regions of almost uniform slope for the splittings. Values of $|d \Delta \nu / d \theta|$ as well as other pertinent characteristics of the $\alpha$ and $\beta$ components are given in table 1 for the three special orientations.

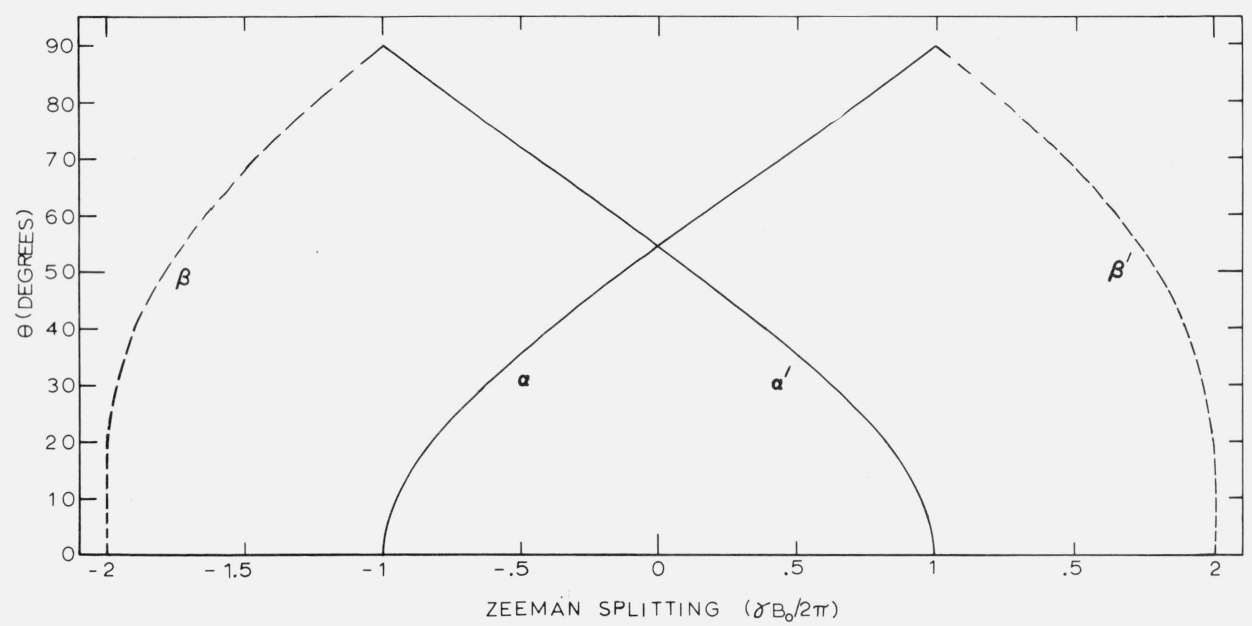

Figure 2. Low-field Zeeman splittings for $|1 / 2| \leftrightarrow|3 / 2|$ transitions in the case $\eta=0$. 


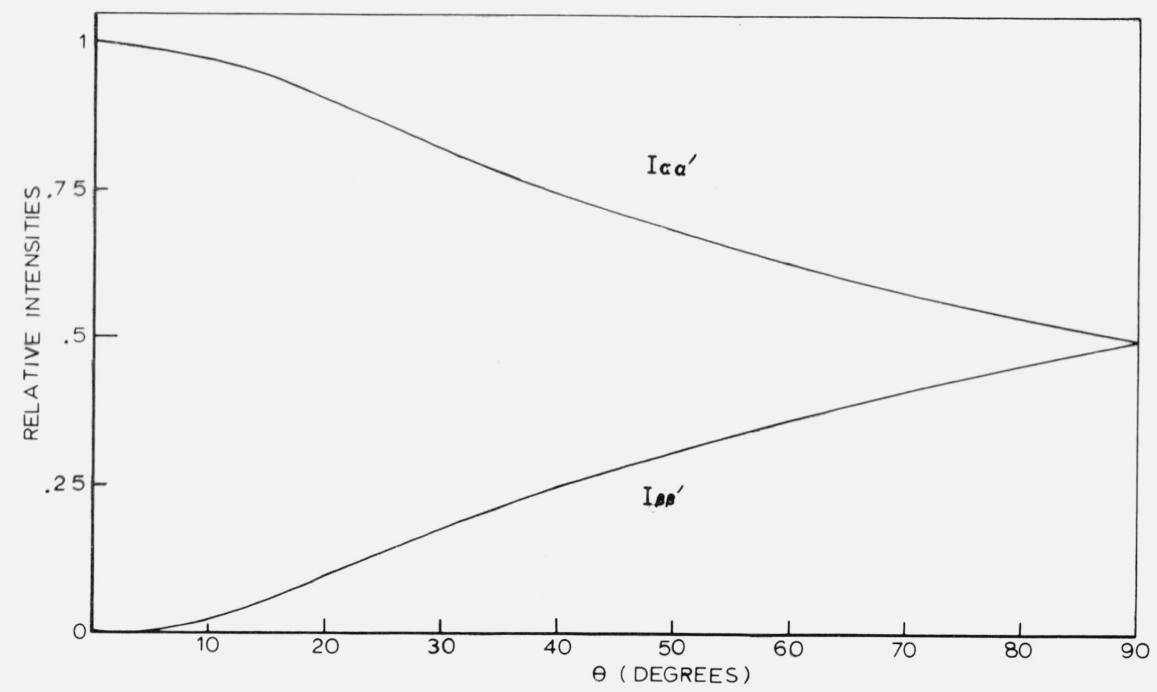

FIGURE 3. Relative intensities of the low-field Zeeman components for $|1 / 2| \leftrightarrow|3 / 2|$ transitions with $\eta=0$.

TABLE 1. Low-field Zeeman parameters of $|1 / 2| \leftrightarrow|3 / 2|$ transitions with $\eta=0$ for three special orientations of the magnetic field with respect to the symmetry axis

\begin{tabular}{|c|c|c|c|c|c|c|}
\hline$\theta$ & \multicolumn{2}{|c|}{$0^{\circ}$} & \multicolumn{2}{|c|}{$54^{\circ} 44^{\prime}$} & \multicolumn{2}{|c|}{$90^{\circ}$} \\
\hline line components... & $\alpha \alpha^{\prime}$ & $\beta \beta^{\prime}$ & $\alpha \alpha^{\prime}$ & $\beta \beta^{\prime}$ & $\alpha \alpha^{\prime}$ & $\beta \beta^{\prime}$ \\
\hline$\Delta \nu\left(\nu_{L}\right) \ldots \ldots \ldots \ldots$ & 2 & 4 & 0 & $2 \sqrt{3}$ & 2 & 2 \\
\hline Relative intensity............ & 1 & 0 & $2 / 3$ & $1 / 3$ & $1 / 2$ & $\cdot 1 / 2$ \\
\hline $\begin{array}{l}\text { Fractional departure of } \Delta \nu \\
\quad \text { from linearity................. }\end{array}$ & 0 & 0 & $\sim \xi^{4 *}$ & $-\xi^{2} / 3$ & $-3 \xi^{2} / 2$ & 0 \\
\hline$|d \Delta \nu / d \theta|\left(\nu_{L} /\right.$ radian $)$ & 0 & 0 & $\sqrt{3}+1$ & $\sqrt{3}-1$ & 3 & 3 \\
\hline
\end{tabular}

*Broadening

A measurement of the $\alpha$ and $\beta$ splittings corresponding to a single orientation of the symmetry axis is sufficient to yield both $\theta$ and $B_{0}$. The field direction is then determined to be somewhere on the surface of a right circular cone making the angle $\theta$ with respect to the field-gradient axis. To ascertain the exact spatial alinement of the field, three non-equivalently oriented symmetry axes are required.

At magnetic fields for which the Zeeman splittings are comparable to or less than the linewidth, the $\alpha$ and $\beta$ components will overlap each other. When the field direction is known, a measurement of the breadth or shape of the composite line will furnish the flux density. In principle, such a determination could be made to a precision approaching that of a measurement of the splitting frequencies in a resolved spectrum. A major disadvantage, however, is the complex dependence of linewidth and lineshape on flux density and the need to calibrate this dependence for the particular crystal under study. An analogous and perhaps more difficult problem would be encountered in the measurement of field direction at low flux densities.

At low fields, special care must be taken to insure that the amplitude of the oscillating rf field, $B_{1}$, is much less than the static field intensity, $B_{0}$. Otherwise, the splittings will be increased [10] by a fractional amount $\left(B_{1} / 2 B_{0}\right)^{2}$.

An nqr magnetometer is incapable of determining the polarity of the magnetic field unless circularly polarized excitation is employed. Depending on the sign of the gyromagnetic ratio and the polarity of the field, this type of $\mathrm{rf}$ field distribution will eliminate the lines arising from transitions involving either pure $+m$ or $-m$ states [11]. Furthermore, the correction term $\left(B_{1} / 2 B_{0}\right)^{2}$ in the Zeeman splittings will be absent, since it arises from the circularly rotating component of a linearly polarized field which is ineffective in producing transitions to or from a particular $m$ state.

Circular polarization also serves to simplify the observed spectrum. In the case of the $|1 / 2| \leftrightarrow|3 / 2|$ transitions, the complex four-line pattern, $\alpha \alpha^{\prime} \beta \beta^{\prime}$ is reduced to either $\alpha, \beta$ or $\alpha^{\prime}, \beta^{\prime}$ components. This is due to the fact that although the $\pm 1 / 2$ states are admixed to zeroth order in the presence of a field, the $\pm 3 / 2$ states remain relatively pure $m$ states as long as $\xi \ll 1$. Thus, as can be seen from figure 2 , the overlap which occurs at angles close to $90^{\circ}$ or $54^{\circ} 44^{\prime}$ is eliminated and improved accuracy results.

Because the $\beta$ components vanish as $\theta$ approaches $0^{\circ}$, measurements of the $\alpha \beta$ splittings made with circularly polarized radiation will be limited to those angles at which the $\beta$ components are sufficiently intense. On the other hand, the $\alpha$ component (and observable $\beta$ component) splittings may be measured separately by switching the direction of polarization or by subjecting two crystals to counter-rotating rf fields. At low fields, these techniques should also make it possible to obtain the splitting between the composite $\alpha, \beta$ and $\alpha^{\prime}, \beta^{\prime}$ 
lines. Thus, the necessity of determining the width or shape of the resonance line, and the corresponding problems associated with the dependence of these parameters on temperature gradients (see below) are avoided. For arbitrary crystal orientation, however, accurate field measurements will still be difficult because the composite lines are in general asymmetrical due to the different intensities of the $\alpha$ and $\beta$ components. This problem does not exist if $\theta$ is equal to $0^{\circ}$ or if the transitions $\pm m \leftrightarrow \pm(m+1)$, with $m \geqslant 1$, are studied. Under these circumstances, only one line will be observed for each direction of polarization, thereby eliminating the overlap and improving the resolution at very low magnetic fields.

\section{The Potassium Chlorate Sensor}

The foregoing discussion demonstrates that Zeeman splittings in nqr can yield information regarding the magnitude and direction of a magnetic field. Thus, in principle, an nqr spectrometer with a properly chosen sample can function as a magnetometer. To examine the usefulness of this technique, we shall consider the case of a single crystal of potassium chlorate. This compound has several desirable features which make it a suitable sensor for this application. The $\mathrm{ClO}_{3}^{-}$ions in the unit cell are completely equivalent with regard to their resonant behavior in an external magnetic field. The unsplit ${ }^{35} \mathrm{Cl}$ resonance line occurs at an experimentally convenient frequency of $28.1 \mathrm{MHz}$ at room temperature and is characterized by an asymmetry parameter close to zero $(\eta \sim 0.005),{ }^{3}$ and one of the narrowest widths of any quadrupole line yet observed [12]. The first derivative curve in the single crystal was found to have a peak-to-peak linewidth of only 0.29 $\mathrm{kHz}$ and exhibited partially resolved Zeeman splittings in fields estimated to be considerably less than earth's field [12].

At $\theta=0^{\circ}$, one set of lines is obtained in $\mathrm{KClO}_{3}$ with a splitting parameter of $\frac{2 \gamma\left({ }^{35} \mathrm{Cl}\right)}{2 \pi}=8.350 \mathrm{kHz} / \mathrm{mT}$. For this orientation, a linewidth of $0.29 \mathrm{kHz}$ is equivalent to $3.5 \mu \mathrm{T}$ in field units. Assuming a measurement of the line center to $1 / 500$ of the linewidth, the splitting of two completely resolved lines in a uniform field at $\theta=0^{\circ}$ could be determined to a precision of approximately $\sqrt{2}(3.5) / 500 \mu \mathrm{T}$, or $10 \mathrm{nT}$. This precision can be achieved at flux densities exceeding $100 \mu \mathrm{T}$ and at lower fields if circularly polarized excitation is employed. Similarly, at $\theta=90^{\circ}$, the limit of detection of changes in angle can be shown to be approximately $\left(65 / B_{0}\right)$ microradians, where $B_{0}$ is expressed in $\mathrm{mT}$.

The uncertainty in the absolute value of the flux density will be determined either by the precision of the measurement or by our knowledge of the gyromagnetic ratio of ${ }^{35} \mathrm{Cl}$ in $\mathrm{KClO}_{3}$. The value of $\gamma\left({ }^{35} \mathrm{Cl}\right)$ used here is taken from a measurement of the nuclear moment in $\mathrm{NaClO}_{3}$ of $0.8215 \pm 0.0001$ nuclear magnetons [9]. This is equivalent to a gyromagnetic ratio of

\footnotetext{
3 This value has been slightly modified from that reported in reference 12 by the use of the correct value of the slope $d \Delta \nu_{\alpha \alpha^{\prime}} / d \theta$ at $\theta=54^{\circ} 44^{\prime}$, rather than the value appropriate to the correct
$\theta=90^{\circ}$.
}

$(\gamma / 2 \pi)=4.175 \pm 0.0005 \mathrm{kHz} / \mathrm{mT}$. It is reasonable to assume that the diamagnetic shielding of the chlorine nucleus in the sodium and potassium compounds is identical, at least to the accuracy given above. The chemical shift anisotropy is also expected to be negligible. For the ${ }^{35} \mathrm{Cl}$ nucleus in $\mathrm{NaClO}_{3}$, Kawamori and Itoh [13] report a fractional difference between the shielding at the $\theta=0^{\circ}$ and $90^{\circ}$ orientations of only $4 \pm 4 \times 10^{-5}$, in good agreement with a calculation neglecting the effect of the cation.

It would be preferable to make a direct measurement of the angular dependence of the gyromagnetic ratio of the ${ }^{35} \mathrm{Cl}$ in $\mathrm{KClO}_{3}$. The ultimate accuracy which can be achieved with the nqr magnetometer will then be determined by the uncertainty in the gyromagnetic ratio of the particular reference sample used to calibrate the magnetic field. At the present time, the primary standard is the gyromagnetic ratio of the proton resonance in liquid water which is known to an accuracy of $\sim 6 \times 10^{-6}$ [14]. This figure also represents the minimum uncertainty attainable with $\mathrm{nmr}$, epr, and Rubidium magnetometers since these too require a measurement of the gyromagnetic ratio of the resonating species used in the sensor.

Next we consider the effect of the small field-gradient asymmetry in $\mathrm{KClO}_{3}$ on the splitting frequencies. Although the value $\eta=0.005$ is only approximate, it will be used in the following calculation to furnish a rough estimate of the deviations from the equations given in section 1 .

At $\theta=0^{\circ}$, exact solutions for the energy levels obtain [9]. The corresponding splitting frequencies are

$$
\begin{aligned}
\Delta \nu_{\alpha \alpha^{\prime}} & =2 \nu_{L} \\
\Delta \nu_{\beta \beta^{\prime}} & =2 \nu_{Q}\left\{\left[(1+2 \xi)^{2}+\eta^{2} / 3\right]^{1 / 2}-\left[(1-2 \xi)^{2}+\eta^{2} / 3\right]^{1 / 2}\right\} \\
& \simeq 4 \nu_{L}\left(1-\eta^{2} / 6\right) \quad(\xi, \eta \ll 1) .
\end{aligned}
$$

The splitting of the $\alpha$ components is exactly the same as in the case of zero asymmetry and shows no departure from linearity with respect to the magnetic field. The correction term for the zero-intensity $\beta$ components is $-\eta^{2} / 6$ or $4 \times 10^{-6}$ for $\mathrm{KClO}_{3}$.

At an arbitrary angle, the frequencies for small $\eta$ are given by the equations [1]

$$
\begin{aligned}
\Delta \nu_{\alpha \alpha^{\prime}}=\nu_{l} \mid\left[\cos ^{2} \theta+\sin ^{2} \theta\right. & \left.\left(4+\eta^{2}-4 \eta \cos ^{2} \phi\right)\right]^{1 / 2} \\
& -\left(9 \cos ^{2} \theta+\eta^{2} \sin ^{2} \theta\right)^{1 / 2} \mid \\
\Delta \nu_{\beta \beta^{\prime}}=\nu_{L}\left\{\left[\cos ^{2} \theta+\sin ^{2} \theta\right.\right. & \left.\left(4+\eta^{2}-4 \eta \cos ^{2} \phi\right)\right]^{1 / 2} \\
& \left.+\left(9 \cos ^{2} \theta+\eta^{2} \sin ^{2} \theta\right)^{1 / 2}\right\}
\end{aligned}
$$

where $\phi$ is the azimuthal angle of the magnetic field referenced to the axis in the principal axis system along which the field gradient has its minimum value.

The deviations of the Zeeman splittings from the zero asymmetry case become more pronounced as $\theta$ approaches $90^{\circ}$. At this angle, the above equations reduce to

$$
\Delta \nu_{\alpha \alpha^{\prime}}=2 \nu_{L}\left[1-\eta / 2(\cos 2 \phi+1)+\eta^{2} / 8\right]
$$




$$
\Delta \nu_{\beta \beta^{\prime}}=2 \nu_{L}\left[1-\eta / 2(\cos 2 \phi-1)+\eta^{2} / 8\right] .
$$

Maximum deviation of the $\alpha$ splitting from the zero asymmetry case occurs at $\phi=0^{\circ}$ and is equal to $-\eta$ or -0.005 for $\mathrm{KClO}_{3}$. At $\phi=90^{\circ}$, the frequency differs from $2 \nu_{L}$ by a negligible amount, $\eta^{2} / 8$. The deviations of the $\beta$ splittings cover the same range, but the maximum in $+\eta$ and occurs at $\phi=90^{\circ}$. Thus if $\phi$ is unknown, the measured value of the magnetic field can be in error by as much as $|\eta|$.

The $\cos 2 \phi$ terms in the above equations may be eliminated by rapidly spinning the crystal about the major axis of the field gradient or by orienting the crystal at $\phi=45^{\circ}$. The effect of a non-zero asymmetry parameter is then determined primarily by the terms $\mp\left(9 \cos ^{2} \theta+\eta^{2} \sin ^{2} \theta\right)^{1 / 2}$ and decreases rapidly with $\theta$. For example, at $\theta=88^{\circ}$, the deviations are approximately equal to $\mp 5 \times 10^{-5}$, down from $\mp 2.5 \times 10^{-3}$ at $\theta=90^{\circ}$. The entire term $\left(9 \cos ^{2} \theta+\eta^{2} \sin ^{2} \theta\right)^{1 / 2}$ can be made to vanish if the $\alpha, \beta$ splittings, $\Delta \nu_{\alpha \beta}$ or $\Delta \nu_{\alpha^{\prime} \beta^{\prime}}$, or the average value of the $\alpha$ and $\beta$ splittings,

$$
\left(\Delta \nu_{\alpha \alpha^{\prime}}+\Delta \nu_{\beta \beta^{\prime}}\right) / 2 \text {, }
$$

are used to measure the field. Indeed, at angles sufficiently close to $90^{\circ}$ to cause appreciable line overlap, it is easier to determine the average than the individual splittings. For the true average to be obtained, however, it is imperative that the composite line be symmetrically split or broadened. This condition implies approximately equal intensities for the $\alpha$ and $\beta$ components at $\theta \simeq 90^{\circ}$ and will occur for $\eta \neq 0$ only if the rf field is alined midway between the two minor axes of the field gradient [4] or if the crystal is rotated rapidly about the major axis. These probe configurations are unnecessary, however, if circular polarization is employed. In this case the $\alpha, \beta$ or $\alpha^{\prime}, \beta^{\prime}$ splittings may be observed directly without overlap except at very low fields.

By combining a measurement of $\Delta \nu_{\alpha \beta\left(\alpha^{\prime} \beta^{\prime}\right)}$ or $\left(\Delta \nu_{\alpha \alpha^{\prime}}+\Delta \nu_{\beta \beta^{\prime}}\right) / 2$ with sample spinning or a crystal alinement of $\phi=45^{\circ}$, the effect of the small asymmetry parameter in $\mathrm{KClO}_{3}$ will be virtually eliminated. The maximum deviation from the zero asymmetry case then occurs at $\theta=90^{\circ}$ and is equal to $\eta^{2} / 8$ or $3 \times 10^{-6}$.

At high fields, terms in $\xi$ and cross-terms in $\xi$ and $\eta$ must be included in the expressions for the splittings. When $\xi \gg \eta$, the departures from linearity with respect to the field may be derived from the equations of Ting, Manring, and Williams [9] for the case $\eta=0$ and have been given in table 1 for the three special orientations. The maximum deviation from linearity is of the order $\xi^{2}$ and is approximately 1 percent at $1 \mathrm{~T}$ for the $\mathrm{KClO}_{3}$ crystal. In the intermediate region, $\xi \sim \eta$, the field dependence of the splittings can be obtained by numerically diagonalizing the Hamiltonian matrix. Closed-form solutions for any value of $\xi$ have been given [7], however, for the special cases $\theta=90^{\circ}$, $\phi=0^{\circ}, 90^{\circ}$.

In conclusion, the simplified equations of section 1 may be applied to the ${ }^{35} \mathrm{Cl}$ spectrum in $\mathrm{KClO}_{3}$ when (1) $\theta$ is equal to $0^{\circ}$, or (2) the field is sufficiently low and the procedures described earlier are taken to elimi- nate the terms in $\eta$. If these conditions are not met and if the simple equations do not yield sufficient accuracy, then corrections due to finite $\eta$ and $\xi$ can be made.

For the $\mathrm{KClO}_{3}$ sensor, departures from linearity with respect to the field are essentially negligible in the region where the nqr magnetometer is expected to have its greatest utility, namely, below $10 \mathrm{mT}$. At these fields, conventional $\mathrm{nmr}$ magnetometers suffer from a very poor signal-to-noise ratio and epr techniques are generally employed for accurate measurements [15]. An nqr magnetometer with $\mathrm{KClO}_{3}$ as the sensor has decided advantages over both the nmr and epr techniques in its ability to sense the direction of the magnetic field and in not requiring major instrumental changes to measure widely different fields. In addition, it can go to much lower fields than is possible with epr magnetometers using currently available sensors, and has much greater accuracy in the low field regime.

\section{Experimental Methods}

Among the outstanding features of the nqr magnetometer is the wide range of magnetic fields that can be measured with essentially a single-frequency spectrometer and one probe, with all the concomitant advantages of convenience, sensitivity, and compactness. For example, with potassium chlorate as the sensor, a $28 \mathrm{-MHz}$ spectrometer with an $8 \mathrm{MHz}$ band width can cover the entire range of roughly $10 \mathrm{nT}$ to $1 \mathrm{~T}$.

Because of the strong temperature dependence of quadrupole frequencies $\left(5.1 \mathrm{kHz} /{ }^{\circ} \mathrm{C}\right.$ for $\mathrm{KClO}_{3}$ at room temperature [16]), an instantaneous recording of the Zeeman splitting to a high degree of precision is desirable. A magnetometer possessing this capability will also make possible the rapid measurement of the direction and magnitude of an unknown field, as well as fast fluctuations in these quantities. One way of accomplishing this is shown schematically in figure 4 . An amplitude- or frequency-modulated spectrometer is employed. The center frequency of the spectrometer is swept.until it locks to the midpoint of one line and the separation of an AM or FM sideband is simultaneously varied until locking is achieved to the other line. The frequency of the low-frequency oscillator which amplitude or frequency modulates the carrier is then locked to, and exactly equals, the Zeeman splitting. Alternatively, a signal-feedback technique may be employed to yield self-oscillation at the splitting frequency.

At low magnetic fields, a linewidth measurement, rather than a splitting frequency, may be required. The locking technique just described can be used to determine this quantity if the resonance curve has at least two zero crossover points symmetrically situated with respect to the line center. The second-derivative absorption or first-derivative dispersion shape function has this property and is easily observed experimentally.

If the circularly polarized configuration is employed at low fields, the splitting may be determined by rapidly switching the direction of polarization in a 


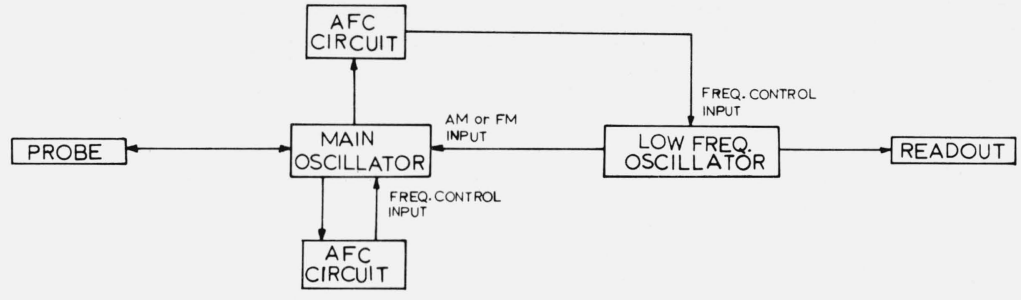

Figure 4. Magnetometer circuit suitable for observation of the instantaneous values of the Zeeman splittings.

time short compared to the time it takes for the sample temperature to drift or the static field to change. Alternatively, two sensors with oppositely rotating circularly polarized fields will give an instantaneous readout of the splitting frequency.

An instantaneous recording of the Zeeman splitting will obviate the necessity of thermostating the sample to a high degree or of accurately determining the temperature at which the measurement was made. Although the pure quadrupole frequency and asymmetry parameter depend strongly on temperature [1], the splitting will be relatively insensitive to temperature if $\eta$ and $\xi$ are much less than one. In the case of $\mathrm{KClO}_{3}$, changes in the vibrational frequencies of the $\mathrm{ClO}_{3}^{-}$ion with temperature will affect both the field gradient and asymmetry parameter to the order of $10^{-4} /{ }^{\circ} \mathrm{C}$ at room temperature. From the equations given earlier, the splittings can be seen to be most sensitive to temperature at the $\theta=90^{\circ}$ orientation. At low fields, the temperature dependence of the $\alpha$ or $\beta$ splittings is at most $d \eta / d \mathrm{~T} \simeq 10^{-4} /{ }^{\circ} \mathrm{C}$ for the static crystal, $\phi \neq 45^{\circ}$, configuration and decreases rapidly as $\theta$ deviates from $90^{\circ}$. At high fields, the splitting frequencies also exhibit a variation with temperature because of their dependence on $\xi^{2}$. For the case $\theta=90^{\circ}$, and $B_{0}=$ IT, the change in the $\alpha$ splitting due to this effect is $3 \xi^{2}\left(d \nu_{Q} / \nu_{Q}\right) d T \simeq 10^{-5} /{ }^{\circ} \mathrm{C}$.

Even if these variations are tolerable, some degree of temperature stabilization is always desirable. In the first place, large temperature instabilities introduce noise in the frequency-locking circuitry and may even cause the system to lose lock. Secondly, a temperature gradient over the sample will broaden the lines and thus adversely affect the precision of the measurement.

Temperature stabilization might also be necessary at low fields when lineshape or linewidth studies are employed for the field measurements. In this case, accuracy as well as precision would suffer from the presence of temperature gradients and changes in the gradients. If the sample is at uniform temperature, however, temperature drifts would have little effect as long as the widths of the individual resonance lines are insensitive to temperature. This condition is satisfied by the ${ }^{35} \mathrm{Cl}$ spectrum in $\mathrm{KClO}_{3}[11,17]$; thus, drifts in sample temperature would change the width of the composite line by a mere $d \eta / d T \simeq 10^{-4} /{ }^{\circ} \mathrm{C}$ in the worst case $\left(\theta=90^{\circ}, \phi=0^{\circ}\right.$ or $\left.90^{\circ}\right)$.
Both the magnitude and direction of a magnetic field can be obtained with a sensor composed of either three orthogonally oriented crystals, one crystal containing at least three nonequivalently oriented symmetry axes with one axis out of the plane of the other two, or variants of these. Alternatively, a single crystal can be mounted on a goniometer assembly and its position adjusted to yield the pattern characteristic of a given orientation.

If changes in field direction are expected to be small, a single crystal configuration with $\theta$ set close to $0^{\circ}$ may suffice in may applications. In this case, the magnetometer would respond to changes in the magnitude of the field but be relatively insensitive to its direction. For the $|1 / 2| \leftrightarrow|3 / 2|$ transitions, this configuration has the additional advantages of furnishing $\alpha$ component signals with maximum intensity and minimum overlap and with splittings which are independent of $\eta, \xi$, and temperature.

Time-dependent variations in the magnitude and direction of a magnetic field can be studied by combining the appropriate sample configuration with the instantaneous recording method outlined above. Sophisticated systems can be developed to give separate digital readouts of both these parameters.

Finally, the precision and range of an nqr magnetometer could be considerably improved upon by the use of line-sharpening techniques. In one such method [18], resolution enhancement is achieved by mixing the resonance line with a suitable combination of its higher derivatives.

\section{References}

[1] Das, J. P., and Hahn, E. L., Nuclear Quadrupole Resonance Spectroscopy, Solid State Physics, Supplement 1 (Academic Press, 1958).

[2] Casabella, P. A., and Bray, P. J., J. Chem. Phys. 28, 1182 (1958).

[3] Casabella, P. A., and Bray, P. J., J. Chem. Phys. 29, 1105 (1958).

[4] Toyama, M., J. Phys. Soc. Japan 14, 1727 (1959).

[5] Morino, Y., and Toyama, M., J. Chem. Phys. 35, 1289 (1961).

[6] Greschishkin, V. S., and Ainbinder, N. E., Sov. Phys.-Solid State, 3, 1325 (1961).

[7] Greschishkin, V. S., and Ainbinder, N. E., Sov. Phys.-Solid State, 3, 2175 (1962).

[8] Negita, H., J. Chem. Phys. 44, 1734 (1966).

[9] Ting, Y., Manring, E. R., and Williams, D., Phys. Rev. 96, 408 (1954). 
[10] Bloch, F., and Siegert, A., Phys. Rev. 57, 522 (1940).

[11] Weber, M. J., and Hahn, E. L., Phys. Rev. 120, 365 (1960).

[12] Zeldes, H., and Livingston, R., J. Chem. Phys. 26, 5 (1957).

[13] Kawamori, A., and Itoh, J., J. Phys. Soc. Japan 18, 1614 (1963).

[14] 12th Report of Advisory Committee on Electricity, BIPM, 1968 (unpublished).

[15] Ingram, D. J. E., Spectroscopy at Radio and Microwave Frequencies, pp. 286-289, 2nd Ed. (Plenum Press, 1967).
[16] Utton, D. B., J. Res. Nat. Bur. Stand. (U.S.), 71A (Phys. and Chem), No. 2, 125 (1967); Utton, D. B., Metrologia 3, 98 (1967).

[17] Weber, M. J., J. Phys. Chem. Solids 17, 267 (1961).

[18] Allen, L. C., Gladney, H. M., and Glarum, S. H., J. Chem. Phys. 40, 3135 (1965); S. H. Glarum, Rev. Sci. Instr. 36, 771 (1965).

(Paper 73C3\&4-290) 